\title{
Abstracts of Papers Presented at the 122nd Meeting of the American Astronomical Society, held 24-28 July 1966 at Ithaca, New York
}

Mass of a Magnetic Star. Helmut A. ABt, Kitt Peak National Observatory, Peter S. Contr AND Armin J. Deutsch, Mount Wilson and Palomar Observatories, ANd George Wallerstein, University of Washington.-The masses of Ap stars are of interest for their possible bearing on the question of whether such stars are on the main sequence for the first or second times. HD 98088 is an Ap star that has been found to be a double-lined spectroscopic binary having a mass ratio (primary to secondary) of 1.34. Eclipses were searched for and not found, indicating an inclination of $\leqslant 80^{\circ}$ and minimum masses of approximately 1.73 and $1.29 \mathfrak{M}_{\odot}$. Despite a classification of FOp for the primary, its hydrogen lines are much stronger than in such a star and the wings of those lines resemble those of an $\mathrm{A} 3 \mathrm{~V}$ star. Assuming a mass for the secondary from the mass-luminosity relation, we find that the primary mass is about $2.6 \mathfrak{M}_{\odot}$, which agrees very well with the mean mass of normal A3V stars. Thus this Ap star has a normal mass for its present position on the main sequence.

This star has the same orbital and magnetic periods, and the rotational period is also closely the same.

Absolute Measurements of the Radio Flux from Cassiopeia $A$ and Taurus $A$ at 3.64 and 1.94 cm. R. J. Allen AND A. H. BARrett, Massachusetts Institute of Technology.-A cornucopia horn reflector with a $6 \mathrm{~m}^{2}$ aperture has been used to make transit observations of the strong radio sources Cassiopeia A and Taurus A during December 1965 and January 1966. The efficiency of the antenna is known from extensive measurements on a pattern range and the results agree well with theoretical calculations. The receivers have been examined for linearity and stability, and the gas-discharge calibration signals have been measured using liquid-nitrogen-cooled terminations. The data have been corrected for waveguide losses, atmospheric attenuation, receiver nonlinearity, source size, and, in the case of Taurus A, for linear polarization.

The results are presented in Table $I$.

TABLE I.

\begin{tabular}{rccc}
\hline & Cassiopeia A & Taurus A & rms error \\
\hline $8.25 \mathrm{GHz}$ & $612^{\mathrm{a}}$ & $563^{\mathrm{a}}$ & $4.8 \%$ \\
$15.50 \mathrm{GHz}$ & 374 & 461 & 5.9 \\
\hline
\end{tabular}

${ }^{2}$ Units of $10^{-26} \mathrm{~W} \mathrm{~m}^{-2} \mathrm{~Hz}^{-1}$.
The mean date for the measurements is 1966.0 in all cases except Cassiopeia $\mathrm{A}$ at $8.25 \mathrm{GHz}$, where it is 1965.9. The data indicate that the spectrum of Cassiopeia $\mathrm{A}$ is well represented by an expression of the form $S_{\nu}=K_{\nu}{ }^{\alpha}$ for a constant spectral index of $\alpha=-0.76 \pm 0.02$, in contradiction to the results of Baars, Mezger, and Wendker (Astrophys. J. 142, $122,1965)$. The data for Taurus agrees fairly well with the lower-frequency measurements, although a constant $\alpha=-0.26 \pm 0.03$ is less convincing than in the case of Cassiopeia A.

The observations were conducted at the Haystack Research Facility of the Lincoln Laboratory, MIT, with the cooperation and assistance of their staff. This work is supported by the National Aeronautics and Space Administration (Grant NsG-419).

Secular Variations in the Flux from Radio Sources at 3.64 and $1.94 \mathrm{~cm}$. R. J. Allen AND A. H. BARRETt, Massachusetts Institute of Technology.-Observations of the quasi-stellar radio sources $3 \mathrm{C} 273$ and $3 \mathrm{C} 279$, and the galaxy Virgo A have been conducted at 8.25 and $15.50 \mathrm{GHz}(\lambda=3.64$ and $1.94 \mathrm{~cm}$, respectively) with the $37-\mathrm{m}$ antenna of the Haystack Research Facility, MIT Lincoln Laboratory. Measurements obtained during the spring and summer of 1965 have been compared with regular monthly observations from late February to early July of 1966 . A small increase in the antenna temperature of Virgo A during June and July 1966 has been traced to unusual distortions of the antenna from temperature gradients in the structure, and we have therefore assumed the flux from Virgo $A$ to be constant. The values taken are 42.5 flux units (one flux unit $=10^{-26} \mathrm{~W} \mathrm{~m}^{-2} \mathrm{~Hz}^{-1}$ ) at 8.25 $\mathrm{GHz}$ and 29.1 units at $15.50 \mathrm{GHz}$. The measured values of flux for the two quasistellar sources for some selected dates are given in Table I $(8.25 \mathrm{GHz}$, relative error in measurements 0.4 flux units rms) and Table II (15.5 GHz, relative error 0.8 units rms).

It is evident that the rapid increase in the $1.94 \mathrm{~cm}$ flux of 3C273 in early 1966 has leveled off, although the source still has an upturned spectrum similar to $3 \mathrm{C} 84$ and 3C279.

TABLE I. Radio flux at $8.25 \mathrm{GHz}$.

\begin{tabular}{ccc}
\hline Date & 3C273 & 3C279 \\
\hline Sept. 18, 1965 & 32.2 & 11.1 \\
April 10, 1966 & 35.2 & 12.3 \\
July 4, 1966 & 38.6 & 13.5 \\
\hline
\end{tabular}

